\title{
Genome Publication Award 2019
}

Established by the Genome editorial board and its publisher, Canadian Science Publishing, the Genome Publication Award recognizes 10 excellent contributions to the journal each year. The award recognizes publications that make substantial and notable contributions to genetics and genomics research. The following papers are recognized.

Evaluating the efficacy of sample collection approaches and DNA metabarcoding for identifying the diversity of plants utilized by nectivorous bats

Christine E. Edwards, Joel F. Swift, Richard F. Lance, Thomas A. Minckley, and Denise L. Lindsay

Genome, 2019, 62(1): 19-29, https://doi.org/10.1139/gen-2018-0102

Expedited assessment of terrestrial arthropod diversity by coupling Malaise traps with DNA barcoding Jeremy R. deWaard, Valerie Levesque-Beaudin, Stephanie L. deWaard, Natalia V. Ivanova, Jaclyn T.A. McKeown, Renee Miskie, Suresh Naik, Kate H.J. Perez, Sujeevan Ratnasingham, Crystal N. Sobel, Jayme E. Sones, Claudia Steinke, Angela C. Telfer, Andrew D. Young, Monica R. Young, Evgeny V. Zakharov, and Paul D.N. Hebert

Genome, 2019, 62(3): 85-95, https://doi.org/10.1139/gen-2018-0093

Preserving the tree of life of the fish family Cyprinidae in Africa in the face of the ongoing extinction crisis

Mariam Adeoba, Solomon G. Tesfamichael, and Kowiyou Yessoufou

Genome, 2019, 62(3): 170-182, https://doi.org/10.1139/gen-2018-0023

Recalibrating the molecular clock for Arctic marine invertebrates based on DNA barcodes

Tzitziki Loeza-Quintana, Christina M. Carr, Tooba Khan, Yash A. Bhatt, Samantha P. Lyon, Paul D.N. Hebert, and Sarah J. Adamowicz

Genome, 2019, 62(3): 200-216, https://doi.org/10.1139/gen-2018-0107

Physical organization of repetitive sequences and chromosome diversity of barley revealed by fluorescence in situ hybridization (FISH)

Siyu Zhang, Minqiu Zhu, Yi Shang, Jiaqi Wang, Dawadundup, Lifang Zhuang, Jinlong Zhang, Chenggen Chu, and Zengjun Qi

Genome, 2019, 62(5): 329-339, https://doi.org/10.1139/gen-2018-0182

Genome-wide transcript analysis of inflorescence development in wheat

Dae Yeon Kim, Min Jeong Hong, and Yong Weon Seo

Genome, 2019, 62(9): 623-633, https://doi.org/10.1139/gen-2018-0200

Altered expression of cell adhesion genes and hybrid male sterility between subspecies of Drosophila pseudoobscura Alwyn Go, Doaa Alhazmi, and Alberto Civetta

Genome, 2019, 62(10): 657-663, https://doi.org/10.1139/gen-2019-0066

The role of dispersal, selection, and timing of sampling on the false discovery rate of loci under selection during geographic range expansion

Paul Mayrand, Élise Filotas, Julian Wittische, and Patrick M.A. James

Genome, 2019, 62(11): 715-727, https://doi.org/10.1139/gen-2019-0004

Signatures of directional selection in a hybrid yeast population

Xiaoguang Sun, Li Ni, Guan Wang, and Xiaohui Zhang

Genome, 2019, 62(11): 749-760, https://doi.org/10.1139/gen-2019-0075

Sex solves Haldane's dilemma

Donal A. Hickey, and G. Brian Golding

Genome, 2019, 62(11): 761-768, https://doi.org/10.1139/gen-2019-0051 